\title{
Inversion of Array Induction Logs and Its Application
}

\author{
Gao Jie ${ }^{1}$, Zhao Aibin ${ }^{2}$, Peng Fei ${ }^{1}$ and Li Hongqi ${ }^{1}$ \\ (1. School of Resources and Information, China University of Petroleum, Beijing 102249, China) \\ (2. Changqing Oilfield Company, PetroChina, Xi'an, Shaanxi 710021, China)
}

\begin{abstract}
With the help of the modified geometrical factor theory, the Marquardt method was used to calculate the true electrical parameters of the formation from array induction logs. The inversion results derived from the assumed model and some practical cases show that the rebuilt formation profile determined by 2 - $\mathrm{ft}$ resolution array induction logs is reasonable when the formation thickness is greater than $1 \mathrm{~m}$, which thus indicates that the inversion method is reliable and can provide quantitative information for the discrimination of oil/gas or water zone.
\end{abstract}

Key words: Array induction log, modified geometrical factor, Marquardt method, inversion

\section{Introduction}

Electrical log data is the basis of the quantitative evaluation of formation fluid saturations. In the conventional logging suite, the combination of dual induction-laterolog 8 (LL8) or micro-spherically focused $\log$ (MSFL), or dual laterolog-LL8 is selected to determine the resistivity profile of permeable formations, because the difference among three curves is the major indication of invasion (Zhang, 1984). In traditional well logging interpretation, the assessment of invasion can be accomplished based on the traditional interpretation charts, which have many limitations. At present, the inversion method is applied to determining the three parameters (i.e., invasion radius, resistivity of the invaded zone, and the true resistivity of the formation) of the step-profile formation (Gao and Jia, 1999; Zhong, et al., 1985). However, because of the vertical and radial heterogeneity of the formation, multiple solutions could be derived from these three resistivity curves.

Nowadays, array induction logging tools, such as Schlumberger AIT, Baker Atlas HDIL, and Halliburton HRAI, are widely used in China's oilfields. CNPC also has manufactured an array induction logging tool. The main problem is that there are few quantitative applications of array induction logs (Zhang, et al., 1997; Zhang and Zhang, 1998). Whereas, the array induction logs have rich information about the formation (Yang and Nie, 2000), and if appropriate formation models and suitable algorithms are selected, we can not only resolve the ill-posed problem of electrical inversion, but also can invert more parameters much closer to real formation conditions. In this paper, the inversion of array induction logs is discussed and its applications are presented.

\section{Fundamental and forward method}

\subsection{Introduction to array induction tool}

Both domestic and foreign companies have their own array induction tools. The framework of tools is basically similar. The hardware-focusing method is abandoned and the tool usually has one transmitting coil and several receiving coils, and, curves of different resolutions and different investigation depths are obtained based on the software-focusing method (Wu, et al., 1997).

In this paper, the processed data came from a Schlumberger AIT tool, which includes a single transmitting coil and eight groups of receiving coils, spacing from 6 in to $6 \mathrm{ft}$ at three frequencies (i.e., $26.325,52.65,105.3 \mathrm{kHz}$, AIT-B) or one frequency (26.325 kHz, AIT-H). The real and imaginary parts of conductivity can be obtained corresponding to multi-frequency and multi-coil pairs. The surface computer system handles the field log data by using the software-focusing method to obtain different resistivity curves with three vertical resolutions $(1,2$ and $4 \mathrm{ft})$ at different investigation depths (10, 20,30,60, and 90 in).

\subsection{Formation model}

Mud invasion can badly affect the response of electrical logging, which can lead to much more complicated interpretation. When the mud invades into the formation, most of the formation water and a fraction of hydrocarbon may be flushed out from near the borehole, the region called the flushed zone. Outside the flushed zone, formation fluids are partly substituted by mud filtrate and the zone between fully mud filtrate saturated section and formation water saturated section, is called the transitional zone. The volume and depth of the invaded zone and the transitional zone is related to the type of drilling mud, formation porosity, formation permeability, pressure drawdown and the time since the formation was drilled. Generally speaking, the lower the formation porosity, the deeper the invasion. The 
undisturbed formation beyond the transitional zone is called the original zone.

Only three parameters can be derived from the conventional three resistivity curves, therefore, the flushed zone and transitional zone are usually called the invaded zone. But AIT tool has 5 log curves corresponding to one vertical resolution, and with the help of the inversion of array induction $\log$, a 5-parameter formation model can be reconstructed as shown Fig. 1, where $R_{\text {xo }}$ is the flushed zone resistivity, $R_{\mathrm{i}}$ is the transitional zone resistivity, $R_{\mathrm{t}}$ is the true resistivity of the original zone; $r_{1}$ is the flushed zone radius, and $r_{2}$ is the transitional zone radius.

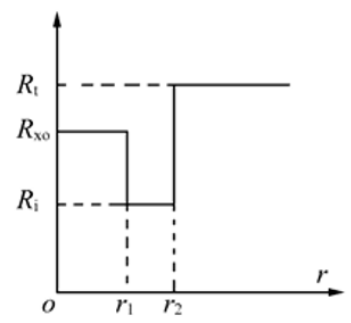

Fig. 1 Schematic diagram of a 5-parameter formation model

\subsection{Forward modeling and configuration of log tools}

Inversion is based on the forward modeling of logging responses and an appropriate forward model method is needed. As for the array induction log, different logging service companies have different software-focusing methods, which usually are proprietary. In the inversion based on array induction logs, the following processing on forward modeling is presented:

1) On the consideration of the inversion speed of resistivity obtained by induction tool, Doll's geometrical factor theory can be applied to forward modeling (Gianero and Anderson, 1982; Moran, 1982). However, Doll's geometrical factor theory neglects skin effects, similar to the synthetic curves that eliminate skin effects during software focusing. Array induction $\log$ forward modeling employing Doll's geometrical factor theory can meet the requirement of increasing calculation speed and enhancing accuracy to some degree.

2) Forward modeling is related to tool configuration. For one vertical resolution of array induction logs, 5 types of equivalent tools are needed to design corresponding 5 different investigation depth curves. In this paper, 2-ft resolution curves of AIT array induction log are used and the equivalent tool only includes two coils. Once the tool configuration is designed, the geometrical factor theory can be used to compute the logging responses.

According to the induction geometrical factor theory and the specific situations of the studied problem, the apparent conductivity can be written in the following form:

$$
\sigma_{\mathrm{a}}=\int_{-\infty}^{\infty} \int_{0}^{\infty} g^{*} \sigma \mathrm{d} r \mathrm{~d} z
$$

with

$$
g^{*}=\beta(\alpha, L) \mathrm{e}^{-\alpha z} g_{\text {Doll }}
$$

where $g^{*}$ is the modified differential geometrical factor of the equivalent tool; $\sigma$ is conductivity; $g_{\text {Doll }}$ is the Doll's differential geometrical factor; $\beta$ is a function of $\alpha$ and $L ; L$ is the spacing of the two-coil system; $\alpha, \beta$ and $L$ is the main parameters of the tool. $g^{*}$, likewise, satisfies:

$$
\int_{-\infty}^{\infty} \int_{0}^{\infty} g^{*} \mathrm{~d} r \mathrm{~d} z=1
$$

There are three consideration in the design of the geometrical factor of the equivalent tool. Firstly, the integration of the geometrical factor $g^{*}$ in the whole space is equal to 1 (i.e., the normalization condition). Secondly, the value of $L$ determines the tool's radial investigation depth. The bigger the value of $L$, the deeper the investigation. Thirdly, the vertical resolution is mainly adjusted by $\alpha$, which has little effect on the radial investigation depth.

For the array induction logging curves with a vertical resolution of $2 \mathrm{ft}$, if $\alpha, \beta$ and $L$ are determined with respect to different investigation depths, a type of equivalent induction log tool is obtained. The values of $\alpha, \beta$ and $L$ at different investigation depths are listed in Table 1. Fig. 2 shows the radial integrated geometrical factor of the equivalent tool, which fits the array induction investigation performance provided by the logging service companies.

Table 1 Equivalent tool parameters corresponding to array induction logs with vertical resolution of $2 \mathrm{ft}$

\begin{tabular}{ccccc}
\hline No & Investigation depth, in & $\alpha$ & $\beta$ & $L, \mathrm{~m}$ \\
\hline 1 & 10 & 2.1 & 1.530 & 0.305 \\
2 & 20 & 6.3 & 4.484 & 0.675 \\
3 & 30 & 7.3 & 7.432 & 1.01 \\
4 & 60 & 2.1 & 14.931 & 1.98 \\
5 & 90 & 2.95 & 22.302 & 2.95 \\
\hline
\end{tabular}

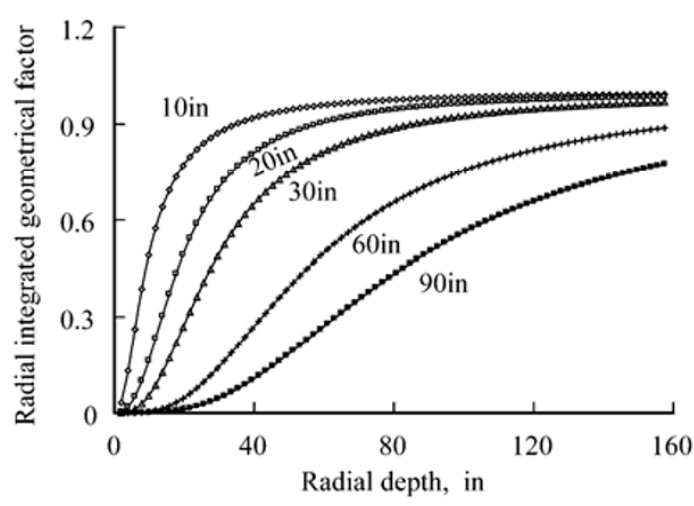

Fig. 2 Radial integrated geometrical factor 


\section{Inversion method}

In the inversion of array induction logs, the Marquardt method is selected as the main inversion method (Gao and Jia, 1999; Hakvoort, 1999). For the Marquardt method, the constructed objective function can be written as follows:

$$
\varphi(\vec{x})=\sum_{k=1}^{m}\left[y_{k}-f_{k}(\vec{x})\right]^{2}
$$

where $y_{k}$ is the measured log; $f_{k}(\vec{x})$ is the computed response from the theoretical model; and $\vec{x}$ is the parameter of the model.

To minimize the objective function, $\frac{\partial \varphi}{\partial \vec{\delta}}=0$ is required, and then the modified quantity $\bar{\delta}$ of $\vec{x}$ can be derived:

$$
\vec{\delta}=A^{-1} \vec{g}
$$

with

$$
\begin{aligned}
& A=P^{\mathrm{T}} P \\
& \vec{g}=P^{\mathrm{T}}\left[\vec{y}-\vec{f}\left(\vec{x}^{(0)}\right)\right]
\end{aligned}
$$

where $P$ is the Jacobi matrix or sensitivity matrix, and its elements are the derivatives of the logging response with respect to each undetermined parameter, which are called Frechet derivative.

The minimization of objective function is to get the inversion results, i.e. the formation parameters.

One disadvantage of the Marquardt method is that its solution usually converges to local minimum points so sometimes the optimum solution can not be obtained. In this paper, limiting the convergence range and selecting better inversion initial value enables the Marquardt method to be used to obtain better results.

\section{Array induction log inversion process and analysis}

\subsection{Method verification and analysis of assumed formation model}

A 5-layer formation model is assumed in this paper. The first and fifth layers are non-permeable formations and others are invaded by mud filtrate. Because of the high vertical resolution of array induction logs, the permeable formations are supposed to be less than $1 \mathrm{~m}$. Details of each formation are shown in Table 2. For the 5-layer formation model, the forward modeling results are displayed in Fig. 3. The inversion results of the parameters of all layers are shown in Table 2, and the

\begin{tabular}{|c|c|c|c|c|c|c|c|c|c|c|c|}
\hline \multirow{2}{*}{ No. } & \multirow{2}{*}{$\begin{array}{c}\text { Thickness } \\
\mathrm{m}\end{array}$} & \multicolumn{2}{|c|}{$R_{\mathrm{x} 0}, \Omega \cdot \mathrm{m}$} & \multicolumn{2}{|c|}{$R_{\mathrm{i}}, \Omega \cdot \mathrm{m}$} & \multicolumn{2}{|c|}{$R_{\mathrm{t}}, \Omega \cdot \mathrm{m}$} & \multicolumn{2}{|c|}{$r_{1}, \mathrm{~m}$} & \multicolumn{2}{|c|}{$r_{2}, \mathrm{~m}$} \\
\hline & & True & Inverted & True & Inverted & True & Inverted & True & Inverted & True & Inverted \\
\hline 1 & 3.0 & 1.0 & 1.08 & 1.0 & 1.08 & 1.0 & 1.08 & 0.1 & 0.1 & 0.1 & 0.1 \\
\hline 2 & 0.6 & 10.0 & 10.49 & 18.0 & 18.23 & 20.0 & 19.73 & 0.3 & 0.31 & 0.6 & 0.81 \\
\hline 3 & 0.7 & 5.0 & 4.05 & 8.0 & 7.54 & 10.0 & 9.89 & 0.4 & 0.39 & 0.8 & 0.76 \\
\hline 4 & 0.5 & 12.0 & 12.75 & 45.0 & 44.74 & 55.0 & 55.23 & 0.3 & 0.31 & 0.9 & 0.88 \\
\hline 5 & 3.0 & 1.0 & 0.94 & 1.0 & 0.937 & 1.0 & 0.937 & 0.1 & 0.1 & 0.1 & 0.1 \\
\hline
\end{tabular}
relative errors of inversion results of each parameter are listed in Table 3.

Table 2 Five-layer formation model parameters and inversion results

Table 3 Relative errors of the inversion results for the 5-layer formation model

\begin{tabular}{cccccc}
\hline No. & Relative error of $R_{\mathrm{xo}}, \%$ & Relative error of $R_{\mathrm{i}}, \%$ & Relative error of $R_{\mathrm{t}}, \%$ & Relative error of $r_{1}, \%$ & Relative error of $r_{2}, \%$ \\
\hline 1 & 8.0 & 8.0 & 8.0 & 0.0 & 0.0 \\
2 & 4.9 & 1.29 & 1.37 & 4.33 & 34.67 \\
3 & 19.0 & 5.7 & 1.08 & 2.0 & 5.5 \\
4 & 6.25 & 0.59 & 0.41 & 1.67 & 2.22 \\
5 & 2.70 & 2.70 & 2.70 & 0.0 & 0.0 \\
\hline
\end{tabular}

If the inversion results are treated as the model parameters and the forward modeling responses are obtained (as shown in Fig. 4). It can be found that the error becomes small, which proves the reasonableness of inversion results to some extent. Specifically, when the permeable formation is thicker than $0.6 \mathrm{~m}$, the formation parameters inversion from $2-\mathrm{ft}$ resolution array induction logs are reliable. 


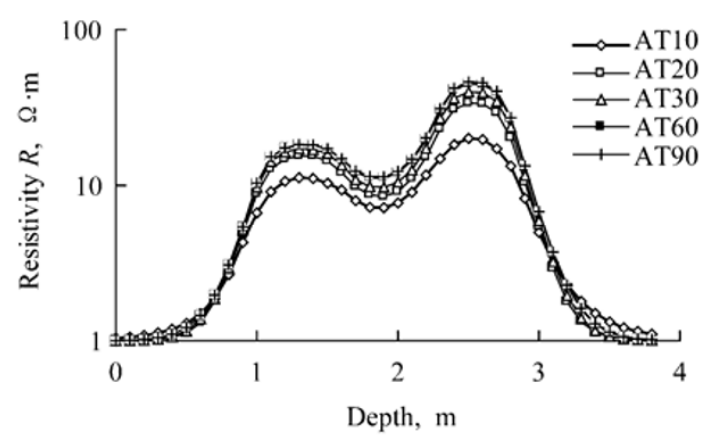

Fig. 3 Five-layer formation modeling responses of true model

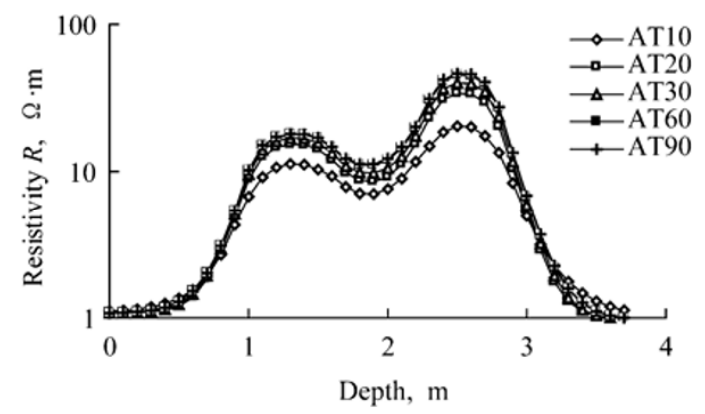

Fig.4 Five-layer formation modeling responses of inversion model

\subsection{Analyses of field logs}

To validate the inversion method, we processed the array induction logs in one oilfield of East China.

The array induction $\operatorname{logs}$ at a depth of 2,407.6-2,412.8 $\mathrm{m}$ is displayed in Fig. 5a. There are distinct differences between these array induction logs. Combined with other well logs, the formation of $2,407.6-2,412.8 \mathrm{~m}$ is determined as a permeable formation by the induction logs. The inversion results of induction logs corresponding to this section are listed in Table 4. This indicates that there exists an obvious low-resistivity annular region at the investigation depth of 2,407.6-2,412.8 m. This region may be an indication of hydrocarbon-bearing formation, which is consistent with the conclusion from log analyst interpretation and oil-testing results.

The measured curves of the interval of 2,302.6-2,305.4 $\mathrm{m}$ in the well are shown in Fig. 5b. The inversion method is applied to interpreting array induction $\log$ for this section, and the inversion results are also shown in Table 4. The inversion results illustrate that the formation was badly invaded and the its undisturbed formation resistivity was lower. Since the well was drilled with fresh mud and the formation salinity is high, it can be concluded that the interval is water-bearing according to highly-resistive invasion, which also agrees with well log analysts' conclusion. All the above validates the reliability of the method proposed in this paper.

Table 4 Inversion results of array induction logs for one well

\begin{tabular}{ccccccc}
\hline Depth, $\mathrm{m}$ & $r_{1}, \mathrm{~m}$ & $r_{2}, \mathrm{~m}$ & $R_{\mathrm{xo}}, \Omega \cdot \mathrm{m}$ & $R_{\mathrm{i}}, \Omega \cdot \mathrm{m}$ & $R_{\mathrm{t}}, \Omega \cdot \mathrm{m}$ & \multicolumn{2}{c}{ Interpretation conclusion } \\
\hline $2407.6-2412.8$ & 0.28 & 0.4 & 38.9 & 8.2 & 37.9 & Oil \\
$2302.6-2305.4$ & 0.16 & 1.99 & 53.81 & 20.77 & 10.18 & Water \\
\hline
\end{tabular}
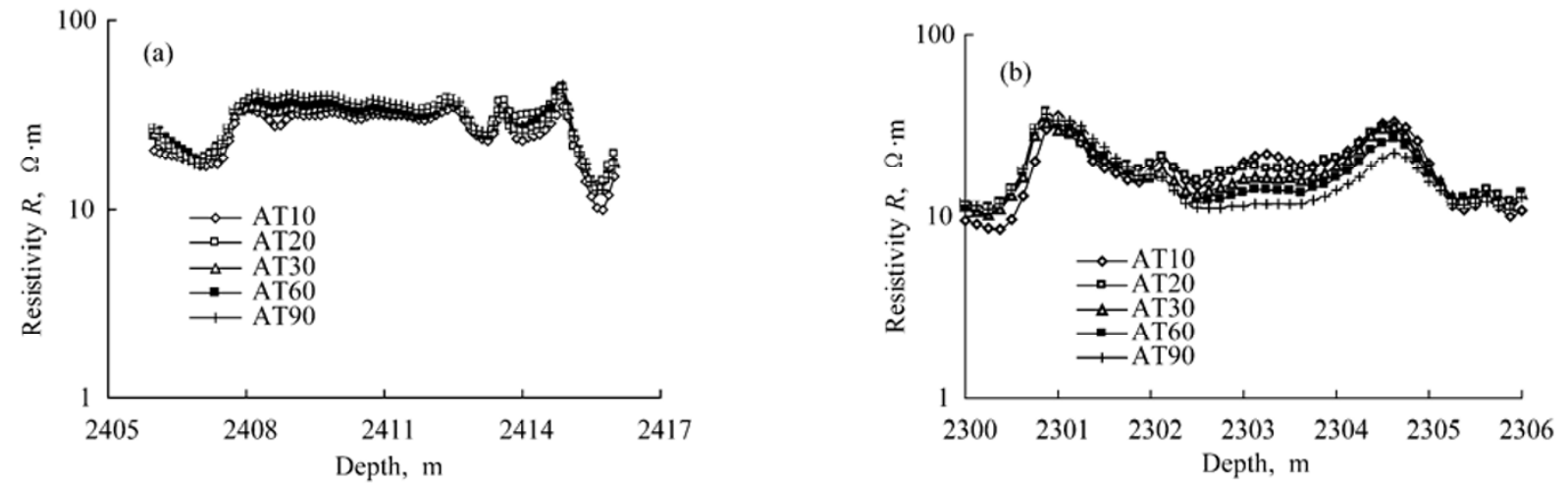

Fig. 5 Array induction logs of one well

\section{Conclusion}

Array induction $\operatorname{logs}$ can provide plentiful information, and the resistivity inversion method is a main approach to utilizing this information quantitatively. The resistivity inversion method can describe the features of mud invasion more clearly and give accurate resistivity of original zones, which could 
be an evidence to identify oil or water section. This paper presents some primary study and application results. The model processing and oil field application show that the results are reliable and the method is feasible. The geometrical factor forward modeling under the equivalent tool configuration is also reliable. Meanwhile, the inversion results of the formation thicker than $1.0 \mathrm{~m}$ indicate that good inversion results can be obtained from array induction logs with a vertical resolution of $2 \mathrm{ft}$. However, boundary determination from the logs is the base of inversion and much remains to be done in this area.

\section{Acknowledgements}

This paper was supported by CNPC Innovation Foundation 2004.

\section{References}

Gao J. and Jia X. P. (1999) Study of dual induction logging resistivity inversion and its application to the Tuha Oilfield. Well Logging Technology, 23(5), 334-337 (in Chinese)

Gianero S. and Anderson B. (1982) A new look at skin effect. The Log Analyst, 23(1), 70-79

Hakvoort R. G. (1999) Inversion of resistivity log. SPWLA 40th Annual Logging Symposium, Oslo, Norway

Moran J. H. (1982) Induction logging: Geometrical factors with skin effect. The Log Analyst, 23(6), 35-39

Wu J., Hu Q. and Wang W. B. (1997) Numerical focusing of array induction measurements and its application. Well Logging Technology, 21(5), 310-317 (in Chinese)
Yang F. and Nie Z. P. (2000) A new inversion method for the formation conductivity profile based on the multi information of Array. Acta Electronic Sinica, 28(6), 135-37

Zhang G. J. (1984) Electrical Well Logging. Beijing: Petroleum Industry Press (in Chinese)

Zhang Y. R., Nie Z. P. and Qi L. F. (1997) A nonlinear inversion approach to formation conductivity: Inversion of AIT measurements. Well Logging Technology, 21(5), 305-309 (in Chinese)

Zhang Z. Q. and Zhang G. J. (1998) Multi parameters inversion of formation resistivity profile using array induction log. Well Logging Technology, 23(5), 321-326 (in Chinese)

Zhong X. S., Huang S. X. and Zhang C. G. (1985) Determine the undisturbed zone resistivity and the invaded zone resistivity by the optimization method. Well Logging Technology, 9(6), 40-45 (in Chinese)

\section{About the first author}

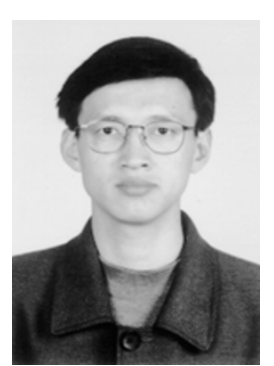

Gao Jie was born in 1969 and received his $\mathrm{PhD}$ degree in applied geophysics from China University of Petroleum in 1996. Now he is an associate professor and works in China University of Petroleum (Beijing), with his research interests in electrical logging theory and data processing. E-mail: jiegao@cup.edu.cn

(Received July 12, 2006) (Edited by Sun Yanhua) 